\title{
Subcarrier Subset Selection Aided Transmit Precoding Achieves Full-Diversity in Index Modulation
}

\author{
Rakshith Rajashekar, Senior Member, IEEE, Chao Xu, Member, IEEE, Naoki Ishikawa, Member, IEEE, \\ Lie-Liang Yang, Fellow, IEEE, and L. Hanzo, Fellow, IEEE
}

\begin{abstract}
Index modulation (IM) is a recently proposed multicarrier transmission scheme, which conveys information both by conventional symbols as well as by the specific subcarrier activation patterns conveying them. However, an impediment of IM is that it lacks transmit diversity gain. In this paper, we circumvent this limitation by proposing a limited-feedback assisted IM transmission scheme. Specifically, Euclidean distance based subcarrier subset selection (ED-SSS) is proposed and its attainable transmit diversity order is shown to be $N_{c}-N_{I M}+1$, where $N_{c}$ is the total number of subcarriers in an IM block and $N_{I M}$ is the number of subcarriers used for IM. Furthermore, the ED-SSS is shown to be amenable to low-complexity implementation owing to the orthogonality of its subcarriers. In order to attain the maximum transmit diversity order of $N_{c}$, ED-SSS is further extended with the aid of transmit precoding and its transmit diversity order is quantified. The proposed precoding assisted ED-SSS is shown to subsume several of the existing precoding aided IM transmission schemes. Simulation studies are conducted for validating our theoretical claims and also for quantifying the attainable performance gains of the proposed schemes. Specifically, at a BER of $10^{-3}$ an SNR gain as high as $8 \mathrm{~dB}$ is observed in case of precoding when compared to its counterpart operating without precoding.
\end{abstract}

Index Terms-Subcarrier subset selection, spatial modulation, index modulation, transmit diversity gain, Euclidean distance.

\section{INTRODUCTION}

Spatial modulation (SM) [1]-[6] is a low-complexity multiantenna transmission scheme capable of attaining a higher energy efficiency [4] than conventional multiple-input multipleoutput (MIMO) transmission schemes [7], [8], [9]. Some of the key advantages of the SM system include: a) A single RFchain at the transmitter [10], [11], resulting in low power dissipation; b) No inter-channel interference (ICI) at the receiver, yielding single-stream low maximum likelihood (ML) detection complexity [12], [13]. Furthermore, SM systems have also been extensively studied in frequency selective fading scenarios [14]-[16], where it was shown to attain a beneficial performance gain over the conventional MIMO schemes at

R. Rajashekar, C. Xu, L-L. Yang, and L. Hanzo are with the School of ECS, University of Southampton, UK (e-mail: rmr1u14@soton.ac.uk, cx1g08@ecs.soton.ac.uk, 1ly@ecs.soton.ac.uk, 1h@ecs.soton.ac.uk).

N. Ishikawa is with the Graduate School of Information Sciences, Hiroshima City University, Japan (e-mail: naoki@ishikawa.cc).

This work was supported in part by the EPSRC projects EP/Noo4558/1 and EP/PO34284/1, the European Research Council's Advanced Fellow Grant under the QuantCom project and the Royal Society's Wolfson Research Merit Award and the GRCF. The work of N. Ishikawa was supported in part by the Japan Society for the Promotion of Science KAKENHI under Grant $17 \mathrm{H} 07036$.
TABLE I

COMPARISON OF VARIOUS EXISTING IM SCHEMES.

\begin{tabular}{l||l}
\hline \multicolumn{1}{|c||}{ Zheng [46] } & \begin{tabular}{l} 
Description \\
\hline \hline Adaptive IM scheme is proposed, which selects the IM pattern \\
consisting of a subcarrier subset and modulation order.
\end{tabular} \\
\hline Choi [48] & $\begin{array}{l}\text { Coded IM scheme is proposed, which employs outer code for } \\
\text { improving the performance of transmitted index bits. }\end{array}$ \\
\hline Zheng \& Chan [49] & $\begin{array}{l}\text { IM scheme is modified to utilize the silent subcarriers } \\
\text { thereby improving the overall performance. }\end{array}$ \\
\hline Gao et al. [50] & $\begin{array}{l}\text { Precoded MIMO-OFDM IM scheme is proposed, } \\
\text { which employs ZF based precoding and selects } \\
\text { the active elements of the receiver side space-frequency blocks. }\end{array}$ \\
\hline Zhang et al. [51] & $\begin{array}{l}\text { Linear precoded IM is proposed, which aims to attain } \\
\text { full transmit diversity by employing Vandermonde } \\
\text { matrix based precoding. }\end{array}$ \\
\hline \hline
\end{tabular}

moderate throughputs. Although, SM is known to offer several benefits over conventional MIMO schemes, it suffers from the lack of transmit diversity gain owing to having a single RF-chain at the transmitter. Several open and closed-loop techniques have been conceived for overcoming this limitation, which includes space-time coded SM transmission schemes [17]-[22], adaptive SM transmission schemes such as a) spatial and signal modulation order selection [23]; b) antenna subset selection [24]-[27] etc. Owing to the high diversity gain [26] of the Euclidean distance based antenna selection (EDAS) scheme [24], it has stimulated a significant research interests [28]-[33]. More specifically, a significant effort has been invested in reducing the computational complexity imposed by the EDAS [24], [28]-[32].

A relative of the SM scheme, namely subcarrier index modulation (IM) [34]-[39] has also gained significant attraction owing to its advantages such as: a) ease of adoption in the existing practical systems; b) superior performance over the conventional orthogonal frequency division multiplexing (OFDM) at moderate throughputs [36]; c) better peak-toaverage power ratio than the conventional OFDM system [40]. Owing to these benefits, IM has been envisioned to be the key technology for the next generation vehicular communications [41]. Analogous to the SM scheme, the IM scheme also suffers from the lack of transmit diversity gain. There are several recent advances in the literature that aim for improving the performance of the IM scheme [42]-[51]. Specifically, Basar 
[42] proposed to achieve a diversity order of two by invoking coordinate interleaving, where the real and imaginary parts of a pair of rotated modulated symbols are swapped before transmission. The associated constellation rotation angle was further optimized by Li et al. [43] based on closed-form analysis, where a precoding based method was also conceived for achieving a diversity order of two. In order to improve the bandwidth efficiency, Wen et al. [44] proposed permutationbased OFDM-IM, where multiple constellation modes were activated in turn in order to increase the number of transmission patterns. In [45], Dang et al. demonstrated that a diversity-throughput tradeoff may be achieved in OFDM-IM, where some subcarrier activation patterns were opportunistically disgarded for better performance. Zheng [46] studied the adaptive modulation order and subcarrier subset selection scheme, where the number of active subcarriers in each IM block is considered to be one. Note that no explicit diversity analysis was presented, although significant performance gains were demonstrated. In [47], Dang et al. further devised an adaptive OFDM-IM scheme for two-hop relaying, where the number of activated subcarriers as well as the associated mapping mechanism were dynamically selected based on the channel quality. In [48], Choi proposed a partially coded IM scheme in order to improve the detection performance of the bits transmitted over the subcarrier indices in the IM block, while transmitting uncoded bits over the conventional symbols. Although, this scheme improves the bit error ratio (BER) performance, it does not increase the overall diversity order of the system. Zheng and Chen [49] proposed an IM scheme that utilizes the inactive subcarriers in the IM block in order to improve the system performance. This scheme was shown to attain a transmit diversity order higher than or equal to two. As a further development, Gao et al. [50] proposed a precoding aided MIMO IM scheme, which eliminates ICI with the aid of a zero-forcing precoder. Although this scheme provides significant performance improvements over the conventional MIMO OFDM system, it requires full channel state information at the transmitter. Recently, Zhang et al. [51] proposed a linearly precoded IM system, which improves the performance of the conventional IM system by exploiting full transmit diversity. Table I summarizes a selection of the above mentioned key diversity-oriented OFDM-IM developments. Against this background, the following are the contributions of this paper:

1) We propose a Euclidean distance based subcarrier subset selection (ED-SSS) scheme, which is the first in the open literature that intrinsically amalgamates the limited-feedback-based subcarrier subset selection (SSS) and index modulation (IM). More explicitly, according to the SSS philosophy, a reduced number of $N_{I M}$ out of $N_{c}$ subcarriers are selected to be utilized based on their associated channel qualities. Furthermore, $N_{a}$ out of $N_{I M}$ are activated based on the IM mapping in order to transmit $N_{a}$ independent complex-valued $M$ ary Phase Shift Keying (PSK) or Quadrature Amplitude Modulation (QAM) symbols. The proposed ED-SSS scheme has the following advantages. First of all, based on limited feedback of the channel quality, the seriously attenuated subcarriers are not used for data transmission. As a result, we prove that the ED-SSS inherently achieves a diversity order of $N_{c}-N_{I M}+1$, which is higher than that of the diversity second-order solutions [42]-[49]. Secondly, we demonstrate that the ED-SSS scheme imposes a low subcarrier selection complexity, which is independent of the constellation size owing to the orthogonality of the subcarriers. This constitutes a key benefit of ED-SSS aided IM, which is not shared by the EDAS aided SM [24], since the spatial signatures are not orthogonal to each other.

2) In order to achieve the maximum attainable transmit diversity, we propose a transmit precoding (TPC) aided ED-SSS scheme, which subsumes the existing precoding based OFDM-IM schemes operating without SSS, such as that of [51]. Based on the classic upper bound of the symbol error probability, we prove that the proposed TPC aided ED-SSS scheme achieves the full diversity order of $N_{c}$. Moreover, the number of subcarrier subset combinations that have to be considered for achieving full diversity is also substantially reduced by the proposed design.

The remainder of the paper is organized as follows. The system model of IM and the ED-SSS are presented in Section II. The low-complexity ED-SSS and its diversity analysis as well as that of the TPC aided ED-SSS scheme are presented in Section III. Our simulation results and discussions are presented in Section IV, while Section V concludes the paper.

Notations: The uppercase boldface letters represent matrices and lowercase boldface letters represent vectors. The notations of $(\cdot)^{T}$ and $(\cdot)^{H}$ indicate the transpose and Hermitian transpose of a vector/matrix, respectively, while $|\cdot|$ represents the cardinality of a given set, or the magnitude of a complex quantity. The notations of $\|\cdot\|$ and $\|\cdot\|_{F}$ represent the two-norm of a vector and the Frobenious norm of a matrix, respectively. $\|\mathbf{x}\|_{0}$ represents the number of non-zero elements in $\mathbf{x} \cdot \operatorname{diag}(\mathbf{x})$ represents a diagonal matrix whose diagonal elements are given by the elements of $\mathbf{x} \cdot \operatorname{bdiag}\left(\mathbf{x}_{1}, \cdots, \mathbf{x}_{N}\right)$ forms a block-wise diagonal matrix based on the component submatrices $\left\{\mathbf{x}_{n}\right\}_{n=1}^{N} .\lfloor x\rfloor$ represents flooring a real-valued number $x$ to the nearest integer. Given a set of indices $I$ and an $n \times n$ matrix $\mathbf{A}, \mathbf{A}(I, I)$ represents a sub-matrix of A whose rows and columns are indexed by the elements of $I$ and $\operatorname{tr}(\mathbf{A})$ represents the trace of $\mathbf{A}$. $\left(\begin{array}{l}m \\ n\end{array}\right)$ represents the number of combinations of choosing $n$ elements out of $m$ elements. $\mathbb{R}$ and $\mathbb{C}$ represent the field of real and complex numbers, respectively. A circularly symmetric complexvalued Gaussian distribution with mean $\beta$ and variance $\sigma^{2}$ is represented by $\mathcal{C N}\left(\beta, \sigma^{2}\right)$. Furthermore, $Q(y)$ represents the tail probability of standard normal distribution given by $\frac{1}{\sqrt{2 \pi}} \int_{y}^{\infty} \exp \left(-\frac{u^{2}}{2}\right) d u$. Expected value of a random variable $X$ is denoted by $\mathrm{E}(X)$. 


\section{PREliminaries}

\section{A. Index Modulation}

Consider a single input multiple output (SIMO) system having $N_{r}$ receive antennas (RAs) and employing IM over $N$ subcarriers. Let the total number of subcarriers be divided into $P$ IM blocks each having $N_{c}$ subcarriers [36]. Let furthermore the number of active subcarriers in each IM block be $N_{a}$. The total number of index activation patterns is given by $\left(\begin{array}{l}N_{c} \\ N_{a}\end{array}\right)$. The IM block in the frequency domain received over $N_{r}$ RAs can be modeled as

$$
\mathbf{y}=\sqrt{\frac{\rho}{N_{a}}} \mathbf{H} \mathbf{x}+\mathbf{n} \in \mathbb{C}^{N_{r} N_{c}},
$$

where $\rho$ is the average signal-to-noise ratio (SNR), $\mathbf{H}=$ $\operatorname{bdiag}\left(\mathbf{h}_{1}, \mathbf{h}_{2}, \ldots, \mathbf{h}_{N_{c}}\right) \in \mathbb{C}^{N_{c} N_{r} \times N_{c}}$ such that $\mathbf{h}_{j} \in \mathbb{C}^{N_{r}}$ is the channel at the $j^{\text {th }}$ subcarrier of the IM block, $\mathbf{x} \in \mathbb{C}^{N_{c}}$ is the transmit IM vector with $N_{a}$ non-zero elements chosen from an $M$-PSK/QAM signal set and $\mathbf{n}$ is the noise vector. The coefficients of $\mathbf{h}_{j}$ for $1 \leq j \leq N_{c}$ and those of $\mathbf{n}$ are modeled as $\mathcal{C N}(0,1)$. Note that the statistically independent channel coefficients over various subcarriers in an IM block can be attained by employing an interleaver [51]. The amount of information conveyed by each IM block is given by

$$
R_{I M}=\left\lfloor\log _{2}\left(\begin{array}{c}
N_{c} \\
N_{a}
\end{array}\right)\right\rfloor+N_{a} \log _{2}(M) \text { bits, }
$$

where $M$ is the size of the QAM/PSK signal set employed. The ML solution assuming perfect channel state information at the receiver (CSIR) is given by

$$
\hat{\mathbf{x}}=\arg \min _{\mathbf{x} \in \mathcal{X}}\left\|\mathbf{y}-\sqrt{\frac{\rho}{N_{a}}} \mathbf{H} \mathbf{x}\right\|^{2},
$$

where $\mathcal{X}$ is the set of all the legitimate transmit IM vectors.

\section{B. Euclidean Distance based Subcarrier Subset Selection}

Assuming that $N_{I M}<N_{c}$ subcarriers are used for IM, there can be a total of $n=\left(\begin{array}{c}N_{c} \\ N_{I M}\end{array}\right)$ possible choices. Let $\mathcal{I}=\left\{I_{1}, I_{2}, \ldots, I_{n}\right\}$ represent the set of enumerations of all possible combinations of $N_{I M}$ subcarriers. Furthermore, let $\Delta \mathcal{X}=\left\{\mathbf{x}_{1}-\mathbf{x}_{2} \mid \mathbf{x}_{1}, \mathbf{x}_{2} \in \mathcal{X}, \mathbf{x}_{1} \neq \mathbf{x}_{2}\right\}$ and $\mathbf{H}_{I}$ represent a submatrix of $\mathbf{H}$, whose columns are indexed by the elements of $I$. The optimal choice of the subcarrier subset based on maximizing the Euclidean distance at the receiver is given by

$$
I^{*}=\arg \max _{I \in \mathcal{I}} \min _{\mathbf{z} \in \Delta \mathcal{X}}\left\|\mathbf{H}_{I} \mathbf{z}\right\|^{2} .
$$

Upon obtaining the optimal subcarrier subset $I^{*}$, the receiver signals the corresponding index to the transmitter over an error free feedback channel. The transmitter then activates the subcarriers indexed by $I^{*}$ for data transmission. It is readily seen that the complexity involved in obtaining $I^{*}$ in (4) is of the order $|\mathcal{I}||\Delta \mathcal{X}|=\left(\begin{array}{c}N_{c} \\ N_{I M}\end{array}\right)\left(\left\lfloor\left(\begin{array}{c}N_{I M} \\ N_{a}\end{array}\right)\right\rfloor M^{N_{a}}\right)^{2}$.

\section{Precoding Aided Subcarrier Subset Selection}

In case of TPC-aided ED-SSS, we have

$$
I^{*}=\arg \max _{I \in \mathcal{I}} \min _{\mathbf{z} \in \Delta \mathcal{X}}\left\|\mathbf{H}_{I} \mathbf{P z}\right\|^{2},
$$

where $\mathbf{P} \in \mathbb{C}^{N_{I M} \times N_{I M}}$ is the TPC matrix that spreads $N_{a}$ symbols across all the $N_{I M}$ subcarriers. The TPC matrix $\mathbf{P}$ has to satisfy $\mathbf{P x} \neq \mathbf{0}$ for all $\mathbf{x} \in \Delta \mathcal{X}$ for unambiguous detection at the receiver. This can be readily ensured by considering a full-rank $\mathbf{P}$, whose null-space constitutes an empty set. Furthermore, the precoded IM symbols are transmitted over the specific subcarriers indexed by $I^{*}$, which enables attaining full transmit diversity, which is a key advantage of TPC, as discussed in detail in the next section.

\section{MAIN RESULTS}

In this section, we first discuss the low-complexity implementation of ED-SSS that exploits the orthogonality of the subcarriers. Furthermore, we quantify the attainable diversity gain of the ED-SSS scheme. Lastly, we discuss the TPCaided ED-SSS, which overcomes the limitation of ED-SSS and attains full transmit as well as the receive diversity gains.

\section{A. Low-Complexity ED-SSS}

Definition 1: A regular $M$-QAM constellation is a Cartesian product of two PAM signal sets, say $M_{1}$-PAM and $M_{2}$ PAM, where $M=M_{1} M_{2}$ and $M_{i}$-PAM $=\left\{-M_{i}+1,-M_{i}+\right.$ $\left.3, \ldots,-1,1, \ldots, M_{i}-3, M_{i}-1\right\}$.

Proposition 1: If $l_{1}, l_{2}, \ldots, l_{N_{c}}$ are the indices of the channel vectors over $N_{c}$ subcarriers such that $\left\|\mathbf{h}_{l_{1}}\right\|^{2}>\left\|\mathbf{h}_{l_{2}}\right\|^{2}>$ $\cdots>\left\|\mathbf{h}_{l_{N_{c}}}\right\|^{2}$ and a regular $M$-QAM constellation is employed over all the $N_{a}$ active subcarriers, then the optimal subcarrier subset in (4) is given by $I^{*}=\left\{l_{1}, l_{2}, \ldots, l_{N_{I M}}\right\}$.

Please refer to Appendix A for the proof. Let us recall that without exploiting the subcarrier orthogonality by Proposition 1, the subset selection of (4) requires the channel state information at the transmitter (CSIT) and has the selection complexity order of $|\mathcal{I}||\Delta \mathcal{X}|=\left(\begin{array}{c}N_{c} \\ N_{I M}\end{array}\right)\left(\left\lfloor\left(\begin{array}{c}N_{I M} \\ N_{a}\end{array}\right)\right\rfloor M^{N_{a}}\right)^{2}$. This is generally the case for the selection in the spatial domain [24], since the spatial signatures are not orthogonal to each other. The precision required for full CSI feedback depends on the target performance. By contrast, the proposed ED-SSS only requires the limited feedback of the channel qualities, where the sorting complexity is on the order of $N_{c} \log N_{c}$, when using algorithms such as Bubble sort, Timsort, Library sort [52]-[54], etc.

Bearing in mind that the PSK signalling has substantial advantages in terms of both BER performance [55],[56] and PAPR [57],[58] in OFDM-IM systems, we offer the following corollary based on Proposition 1:

Corollary 1: When $M$-PSK is employed in ED-SSS aided IM, the optimal subcarrier subset in (4) is also given by $I^{*}=$ $\left\{l_{1}, l_{2}, \ldots, l_{N_{I M}}\right\}$ for $\left\|\mathbf{h}_{l_{1}}\right\|^{2}>\left\|\mathbf{h}_{l_{2}}\right\|^{2}>\cdots>\left\|\mathbf{h}_{l_{N_{c}}}\right\|^{2}$.

Let us consider the example of $M=2$ and $\left|Q^{c}\right|=N_{a}$. We have $\min _{\mathbf{z} \in \Delta \mathcal{X}} \sum_{i \in I}\left\|\mathbf{h}_{i}\right\|^{2}|\mathbf{z}(i)|^{2}=4\left\|\mathbf{h}_{m_{N_{I M}}}\right\|^{2}$. When

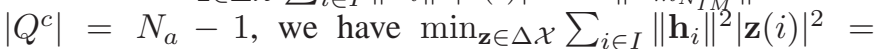
$\left\|\mathbf{h}_{m_{N_{I M}}-1}\right\|^{2}+\left\|\mathbf{h}_{m_{N_{I M}}}\right\|^{2}$. When $\left|Q^{c}\right|=N_{a}-2$, we have $\min _{\mathbf{z} \in \Delta \mathcal{X}} \sum_{i \in I}\left\|\mathbf{h}_{i}\right\|^{2}|\mathbf{z}(i)|^{2}=\sum_{i=0}^{3}\left\|\mathbf{h}_{m_{N_{I M}}-i}\right\|^{2}$. Since $\sum_{i \in I}\left\|\mathbf{h}_{i}\right\|^{2}|\mathbf{z}(i)|^{2}=f\left(\left\|\mathbf{h}_{m_{1}}\right\|^{2},\left\|\mathbf{h}_{m_{2}}\right\|^{2}, \cdots,\left\|\mathbf{h}_{m_{N_{I M}}}\right\|^{2}\right)$ is a linear function for various values of $\left|Q^{c}\right|$, the subset $I^{*}=$ $\left\{l_{1}, l_{2}, \ldots, l_{N_{I M}}\right\}$ is the solution of the optimization problem 
$\max _{I \in \mathcal{I}} \min _{\mathbf{z} \in \Delta \mathcal{X}} \sum_{i \in I}\left\|\mathbf{h}_{i}\right\|^{2}|\mathbf{z}(i)|^{2}$. Similar arguments also hold for the case of $M>2$.

\section{B. Diversity Analysis of ED-SSS}

The system model in (1) can be equivalently written as

$$
\mathbf{Y}=\sqrt{\frac{\rho}{N_{a}}} \mathbf{H}_{s} \mathbf{X}+\mathbf{N} \in \mathbb{C}^{N_{r} \times N_{c}},
$$

where $\mathbf{H}_{s}=\left[\mathbf{h}_{1}, \mathbf{h}_{2}, \ldots, \mathbf{h}_{N_{c}}\right] \in \mathbb{C}^{N_{r} \times N_{c}}, \mathbf{X}=\operatorname{diag}(\mathbf{x}) \in$ $\mathbb{C}^{N_{c} \times N_{c}}$ and $\mathbf{N}$ is the noise matrix. Let $G_{i}$ represent the set of IM indices associated with the subcarrier subset $I_{i}$ for $1 \leq$ $i \leq n$. Let the transmit codebook associated with $I_{i}$ be

$$
\mathcal{C}_{i}=\left\{\operatorname{diag}\left(\sum_{k \in J} s_{k} \mathbf{e}_{k}\right) \mid s_{k} \in M-\mathrm{QAM}, J \in G_{i}\right\} .
$$

Let the set of difference matrices associated with the codebook $\mathcal{C}_{i}$ be

$$
\Delta \mathcal{C}_{i}=\left\{\mathbf{X}-\mathbf{X}^{\prime} \mid \mathbf{X}, \mathbf{X}^{\prime} \in \mathcal{C}_{i}, \mathbf{X} \neq \mathbf{X}^{\prime}\right\} .
$$

Furthermore, let $\mathbf{X}_{\min }(k)=\arg \min _{\mathbf{X} \in \Delta \mathcal{c}_{k}}\left\|\mathbf{H}_{s} \mathbf{X}\right\|_{F}^{2}$, $\mathbf{X}_{\text {min }}=\left[\mathbf{X}_{\min }(1), \mathbf{X}_{\min }(2), \ldots, \mathbf{X}_{\min }(n)\right] \in \mathbb{C}^{N_{c} \times n N_{c}}$ and the index of the optimal subcarrier subset $k^{*}=$ $\arg \max _{l}\left\{\alpha_{l}\right\}_{l=1}^{n}$, where $\alpha_{l}=\min _{\mathbf{X} \in \Delta \mathcal{C}_{l}}\left\|\mathbf{H}_{s} \mathbf{X}\right\|_{F}^{2}$.

Proposition 2: The union bound on the symbol error probability of the ED-SSS is given by

$$
\mathrm{P}_{\mathrm{e}} \leq \frac{\left|\mathcal{C}_{k^{*}}\right|-1}{2}\left(\frac{\rho \lambda^{*}}{4 n N_{a}}\right)^{-q N_{r}},
$$

where $q=\operatorname{rank}\left(\mathbf{X}_{\min }\right)$ and $\lambda^{*}$ is the smallest non-zero Eigenvalue of $\mathbf{X}_{\min } \mathbf{X}_{\min }^{H}$.

Proof: Given the channel realization $\mathbf{H}$, the pairwise error probability (PEP) between any two distinct transmit signals indexed by $m_{1}, m_{2}$ in $\mathcal{C}_{k^{*}}$ is given by

$$
\begin{aligned}
\operatorname{PEP}\left(\mathbf{X}_{m_{1}} \rightarrow \mathbf{X}_{m_{2}} \mid \mathbf{H}_{s}\right) & =Q\left(\sqrt{\frac{\rho}{N_{a}}} \frac{\left\|\mathbf{H}_{s}\left(\mathbf{X}_{m_{1}}-\mathbf{X}_{m_{2}}\right)\right\|_{F}}{2}\right), \\
& \leq \frac{1}{2} \exp \left(\frac{-\rho\left\|\mathbf{H}_{s}\left(\mathbf{X}_{m_{1}}-\mathbf{X}_{m_{2}}\right)\right\|_{F}^{2}}{4 N_{a}}\right) .
\end{aligned}
$$

We note that Craig's formula was invoked for evaluating the exact expression of the $Q$-function in [59]. Nonetheless, in this work, we are mainly concerned with determining the diversity order with the aid of the classic upper bound expression of the PEP following the derivations in [60], [61]. As a result, we have:

$$
\left\|\mathbf{H}_{s}\left(\mathbf{X}_{m_{1}}-\mathbf{X}_{m_{2}}\right)\right\|_{F}^{2} \geq\left\|\mathbf{H}_{s} \mathbf{X}_{m i n}\left(k^{*}\right)\right\|_{F}^{2} \geq \frac{\left\|\mathbf{H}_{s} \mathbf{X}_{m i n}\right\|_{F}^{2}}{n} .
$$

Furthermore, we have $\left\|\mathbf{H}_{s} \mathbf{X}_{\text {min }}\right\|_{F}^{2}=$ $\operatorname{tr}\left(\mathbf{H}_{s} \mathbf{X}_{\min } \mathbf{X}_{\min }^{H} \mathbf{H}_{s}^{H}\right)=\operatorname{tr}\left(\overline{\mathbf{H}}_{s} \mathbf{D}_{\min } \overline{\mathbf{H}}_{s}^{H}\right)$, where $\mathbf{X}_{\text {min }} \mathbf{X}_{\text {min }}^{H}=\mathbf{U D}_{\min } \mathbf{U}^{H}$ and $\overline{\mathbf{H}}_{s}=\mathbf{H}_{s} \mathbf{U}$. Thus, we have $\left\|\mathbf{H}_{s} \mathbf{X}_{\text {min }}\right\|_{F}^{2}=\sum_{i=1}^{N_{r}} \sum_{j=1}^{q} \lambda_{j}\left|\bar{h}_{s}(i, j)\right|^{2} \geq$ $\lambda^{*} \sum_{i=1}^{N_{r}} \sum_{j=1}^{q}\left|\bar{h}_{s}(i, j)\right|^{2}$, where $q=\operatorname{rank}\left(\mathbf{X}_{\text {min }}\right)$ and $\lambda^{*}$ is the smallest non-zero Eigenvalue of $\mathbf{X}_{m i n} \mathbf{X}_{m i n}^{H}$. From the above equations, we arrive at

$\operatorname{PEP}\left(\mathbf{X}_{m_{1}} \rightarrow \mathbf{X}_{m_{2}} \mid \mathbf{H}_{s}\right) \leq \frac{1}{2} \exp \left(\frac{-\rho \lambda^{*}}{4 n N_{a}} \sum_{i=1}^{N_{r}} \sum_{j=1}^{q}\left|\bar{h}_{s}(i, j)\right|^{2}\right)$.

Taking expectation over $\mathbf{H}_{s}$ of (6), we have

$$
\begin{aligned}
\operatorname{PEP}\left(\mathbf{X}_{m_{1}} \rightarrow \mathbf{X}_{m_{2}}\right) & \leq \frac{1}{2} \prod_{i=1}^{N_{r}} \prod_{j=1}^{q} \mathrm{E}\left(\exp \left(\frac{-\rho \lambda^{*}}{4 n N_{a}}\left|\bar{h}_{s}(i, j)\right|^{2}\right)\right) \\
& \leq \frac{1}{2} \prod_{i=1}^{N_{r}} \prod_{j=1}^{q}\left(1+\frac{\rho \lambda^{*}}{4 n N_{a}}\right)^{-1}
\end{aligned}
$$

At high SNRs, we have $\frac{\rho \lambda^{*}}{4 n N_{a}} \gg 1$ and hence

$$
\operatorname{PEP}\left(\mathbf{X}_{m_{1}} \rightarrow \mathbf{X}_{m_{2}}\right) \leq \frac{1}{2}\left(\frac{\rho \lambda^{*}}{4 n N_{a}}\right)^{-q N_{r}} .
$$

Thus, the union bound on the symbol error probability is given by

$$
\begin{aligned}
\mathrm{P}_{\mathrm{e}} & \leq \frac{1}{\left|\mathcal{C}_{k^{*}}\right|} \sum_{\mathbf{X}_{m_{1}} \in \mathcal{C}_{k^{*}}} \sum_{\mathbf{X}_{m_{1}} \neq \mathbf{X}_{m_{2}} \in \mathcal{C}_{k^{*}}} \operatorname{PEP}\left(\mathbf{X}_{m_{1}} \rightarrow \mathbf{X}_{m_{2}}\right) \\
& \leq \frac{\left|\mathcal{C}_{k^{*}}\right|-1}{2}\left(\frac{\rho \lambda^{*}}{4 n N_{a}}\right)^{-q N_{r}}
\end{aligned}
$$

It is evident from (18) that the attainable transmit diversity order of ED-SSS is given by $q=\operatorname{rank}\left(\mathbf{X}_{\text {min }}\right)$. The following proposition states that the transmit diversity order of ED-SSS is $N_{c}-N_{I M}+1$.

Proposition 3: For any given channel realization $\mathbf{H}$, we have $\operatorname{rank}\left(\mathbf{X}_{\text {min }}\right)=N_{c}-N_{I M}+1$ and hence the transmit diversity order of ED-SSS is $N_{c}-N_{I M}+1$.

Proof: Let the set of indices $l_{1}^{(k)}, l_{2}^{(k)}, \ldots, l_{N_{I M}}^{(k)}$ associated with the codebook $\mathcal{C}_{k}$ is such that $\left\|\mathbf{h}_{l_{1}^{(k)}}\right\|^{2}>\left\|\mathbf{h}_{l_{2}^{(k)}}\right\|^{2}>$ $\cdots>\left\|\mathbf{h}_{l_{N_{I M}(k)}}\right\|^{2}$. From Proposition 1, we have $\mathbf{X}_{\min }(k)=$ $4\left\|\mathbf{h}_{l_{N_{I M}(k)}}\right\|^{2} \mathbf{e}_{l_{N_{I M}}^{(k)}} \mathbf{e}_{l_{N_{I M}}^{(k)}}^{T}$, which is a rank-1 matrix. The index $l_{N_{I M}}^{(k)}$ appears in $\left(\begin{array}{c}N_{c}-1 \\ N_{I M-1}\end{array}\right)$ combinations out of $n$ combinations. Thus, $\left(\begin{array}{c}N_{c}-1 \\ N_{I M}-1\end{array}\right) \mathbf{X}_{\text {min }}(i)$ matrices in $\mathbf{X}_{\min }$ belong to the same subspace constituting a rank-1 matrix. Similarly, the next $\left(\begin{array}{c}N_{c}-2 \\ N_{I M}-1\end{array}\right)$ combinations also constitute a set of rank-1 matrices which results in rank-2 $\mathbf{X}_{\min }$. Since there can be $N_{c}-N_{I M}+1$ partitions of $\mathbf{X}_{m i n}$, each constituting a rank1 matrix and linearly independent of the matrices from other partitions, we have $\operatorname{rank}\left(\mathbf{X}_{\min }\right)=N_{c}-N_{I M}+1$.

The following examples illustrate the above concepts.

Example 1: Let $N_{c}=3, N_{I M}=2$ and $N_{r}=1$. Without loss of generality, let $\left|h_{1}\right|^{2}>\left|h_{2}\right|^{2}>\left|h_{3}\right|^{2}$ and

$$
\mathcal{I}=\{\underbrace{\{1,2\}}_{I_{1}}, \underbrace{\{2,3\}}_{I_{2}}, \underbrace{\{1,3\}}_{I_{3}}\} \text {. }
$$


Then, we have

$$
\mathbf{X}_{\text {min }}=\left[\mathbf{X}_{\text {min }}(1) \mathbf{X}_{\text {min }}(2) \mathbf{X}_{\text {min }}(3)\right],
$$

where

$$
\mathbf{X}_{\min }(i)=\left[\begin{array}{lll}
0 & 0 & 0 \\
0 & 0 & 0 \\
0 & 0 & *
\end{array}\right]
$$

for $i \in\{2,3\}$ and

$$
\mathbf{X}_{\min }(1)=\left[\begin{array}{lll}
0 & 0 & 0 \\
0 & * & 0 \\
0 & 0 & 0
\end{array}\right]
$$

It is then readily seen that $\operatorname{rank}\left(\mathbf{X}_{\text {min }}\right)=2$.

Example 2: Let $N_{c}=4, N_{I M}=3$ and $N_{r}=1$. Without loss of generality, let $\left|h_{1}\right|^{2}>\left|h_{2}\right|^{2}>\left|h_{3}\right|^{2}>\left|h_{4}\right|^{2}$ and

$$
\mathcal{I}=\{\underbrace{\{1,2,4\}}_{I_{1}}, \underbrace{\{1,3,4\}}_{I_{2}}, \underbrace{\{2,3,4\}}_{I_{3}}, \underbrace{\{1,2,3\}}_{I_{4}}\} .
$$

Then, we have

$$
\mathbf{X}_{\min }=\left[\mathbf{X}_{\min }(1) \mathbf{X}_{\min }(2) \mathbf{X}_{\min }(3) \mathbf{X}_{\min }(4)\right],
$$

where

$$
\mathbf{X}_{\min }(i)=\left[\begin{array}{cccc}
0 & 0 & 0 & 0 \\
0 & 0 & 0 & 0 \\
0 & 0 & 0 & 0 \\
0 & 0 & 0 & *
\end{array}\right]
$$

for $1 \leq i \leq 3$ and

$$
\mathbf{X}_{\min }(4)=\left[\begin{array}{llll}
0 & 0 & 0 & 0 \\
0 & 0 & 0 & 0 \\
0 & 0 & * & 0 \\
0 & 0 & 0 & 0
\end{array}\right]
$$

Observe that we have $\operatorname{rank}\left(\mathbf{X}_{\min }\right)=2$.

\section{Attaining Full Diversity with Precoding}

Let the transmit codebook associated with $I_{i}$ when employing TPC be

$$
\mathcal{C}_{i}^{\prime}=\left\{\operatorname{diag}\left(\mathbf{P}_{i} \sum_{k \in J} s_{k} \mathbf{e}_{k}\right) \mid s_{k} \in M-\mathrm{QAM}, J \in G_{i}\right\},
$$

where $\mathbf{P}_{i} \in \mathbb{C}^{N_{c} \times N_{c}}$ is the TPC matrix associated with the index subset $I_{i}$, i.e. $\mathbf{P}_{i}\left(I_{i}, I_{i}\right)=\mathbf{P}$ and zeros elsewhere. Let the set of difference matrices associated with the codebook $\mathcal{C}_{i}^{\prime}$ be

$$
\Delta \mathcal{C}_{i}^{\prime}=\left\{\mathbf{X}-\mathbf{X}^{\prime} \mid \mathbf{X}, \mathbf{X}^{\prime} \in \mathcal{C}_{i}^{\prime}, \mathbf{X} \neq \mathbf{X}^{\prime}\right\} .
$$

Furthermore, let $\mathbf{X}_{\min }^{\prime}(k)=\arg \min _{\mathbf{X} \in \Delta \mathcal{C}_{k}^{\prime}}\left\|\mathbf{H}_{s} \mathbf{X}\right\|_{F}^{2}$, $\mathbf{X}_{\text {min }}^{\prime}=\left[\mathbf{X}_{\text {min }}^{\prime}(1), \mathbf{X}_{\min }^{\prime}(2), \ldots, \mathbf{X}_{\min }^{\prime}(n)\right] \in \mathbb{C}^{N_{c} \times n N_{c}}$ and the index of the optimal subcarrier subset be $k^{*}=$ $\arg \max _{l}\left\{\beta_{l}\right\}_{l=1}^{n}$, where $\beta_{l}=\min _{\mathbf{X} \in \Delta \mathcal{C}_{l}^{\prime}}\left\|\mathbf{H}_{s} \mathbf{X}\right\|_{F}^{2}$.

Proposition 4: For any given channel realization $\mathbf{H}$, the transmit diversity order of TPC-aided ED-SSS is $N_{c}$, when $\|\mathbf{P x}\|_{0}=N_{I M} \forall \mathbf{x} \in \Delta \mathcal{X}$.
TABLE II

COMPARISON OF ED-SSS WITH THE EXISTING SCHEMES.

\begin{tabular}{|l|c|c|c|}
\hline & $\begin{array}{c}\text { Channel } \\
\text { signatures }\end{array}$ & Complexity & $\begin{array}{c}\text { Diversity } \\
\text { order }\end{array}$ \\
\hline \hline $\begin{array}{l}\text { GSM/SM with } \\
\text { EDAS [24], [27] }\end{array}$ & Non-orthogonal & $\mathcal{O}\left(M^{N_{a}}\right)$ & $N_{c}-N_{I M}+1$ \\
\hline ED-SSS & Orthogonal & $\begin{array}{c}\text { Independent } \\
\text { of } M\end{array}$ & $N_{c}-N_{I M}+1$ \\
\hline $\begin{array}{l}\text { ED-SSS with } \\
\text { precoding }\end{array}$ & Non-orthogonal & $\mathcal{O}\left(M^{N_{a}}\right)$ & $N_{c}$ \\
\hline \hline
\end{tabular}

Proof: From Proposition 2, it is sufficient to show that $\operatorname{rank}\left(\mathbf{X}_{m i n}^{\prime}\right)=N_{c}$ in order to prove that the transmit diversity order of TPC-aided ED-SSS is $N_{c}$. Note that each $\mathbf{X}_{\min }^{\prime}(k)$ is a diagonal matrix with $N_{I M}$ non-zero elements at locations indexed by $I_{k}$ due to the condition that $\|\mathbf{P x}\|_{0}=N_{I M}$ $\forall \mathbf{x} \in \Delta \mathcal{X}$. Since there exists $I_{1}^{\prime}, I_{2}^{\prime}, \ldots, I_{k}^{\prime}(k \leq n)$ such that $\cup_{i=1}^{k} I_{i}^{\prime}=\left\{1,2, \ldots, N_{c}\right\}$, we have $\operatorname{rank}\left(\mathbf{X}_{\min }^{\prime}\right)=N_{c}$.

Corollary 1: In an IM system employing TPC-aided EDSSS, where the TPC $\mathbf{P}$ satisfies the condition $\|\mathbf{P x}\|_{0}=N_{I M}$ $\forall \mathrm{x} \in \Delta \mathcal{X}$, no more than $\left\lceil\frac{N_{c}}{N_{I M}}\right\rceil$ subcarrier subsets have to be considered for obtaining the optimal subcarrier subset that achieves full transmit diversity gain.

When $N_{c}=N_{I M}$, having a single subcarrier subset suffices in order to attain full transmit diversity gain. This case corresponds to the linearly precoded IM scheme of [51].

Example 3: Let $N_{c}=4, N_{I M}=3, N_{r}=1$ and

$$
\mathcal{I}=\{\underbrace{\{1,2,4\}}_{I_{1}}, \underbrace{\{1,3,4\}}_{I_{2}}, \underbrace{\{2,3,4\}}_{I_{3}}, \underbrace{\{1,2,3\}}_{I_{4}}\}
$$

as in the previous example. Then, we have

$$
\mathbf{X}_{m i n}^{\prime}=\left[\mathbf{X}_{m i n}^{\prime}(1) \mathbf{X}_{m i n}^{\prime}(2) \mathbf{X}_{m i n}^{\prime}(3) \mathbf{X}_{m i n}^{\prime}(4)\right],
$$

where

$\mathbf{X}_{\min }(1)=\left[\begin{array}{cccc}* & 0 & 0 & 0 \\ 0 & * & 0 & 0 \\ 0 & 0 & 0 & 0 \\ 0 & 0 & 0 & *\end{array}\right], \mathbf{X}_{m i n}(2)=\left[\begin{array}{cccc}* & 0 & 0 & 0 \\ 0 & 0 & 0 & 0 \\ 0 & 0 & * & 0 \\ 0 & 0 & 0 & *\end{array}\right]$,

$\mathbf{X}_{\min }(3)=\left[\begin{array}{llll}0 & 0 & 0 & 0 \\ 0 & * & 0 & 0 \\ 0 & 0 & * & 0 \\ 0 & 0 & 0 & *\end{array}\right], \mathbf{X}_{\min }(4)=\left[\begin{array}{cccc}* & 0 & 0 & 0 \\ 0 & * & 0 & 0 \\ 0 & 0 & * & 0 \\ 0 & 0 & 0 & 0\end{array}\right]$

It is then readily seen that $\operatorname{rank}\left(\mathbf{X}_{\min }\right)=4$. Furthermore, it is straightforward to see that any two distinct subcarrier subsets $I_{1}^{\prime}, I_{2}^{\prime}$ yield $I_{1}^{\prime} \cup I_{2}^{\prime}=\{1,2,3,4\}$. Thus, any two distinct subcarrier subset combinations yield full diversity gain.

Table II compares some of the characteristics of the ED-SSS with that of the existing schemes. Note that although TPC in ED-SSS allows achieving full diversity gain, it imposes high computational complexity owing to the non-orthogonal channel signatures. 


\section{Performance under Correlated Subcarriers}

So far we have assumed that the channel coefficients across subcarriers are uncorrelated and statistically independent. Although this condition can be approached by employing a long frequency-domain interleaver [51], the validity of this assumption may become eroded, when we have a limited the number of multipath components. This renders the subcarriers in an IM block correlated. Thus, it is worth extending our analysis to correlated channel conditions. Let the correlated channel over the subcarriers be modeled as $\mathbf{H}_{s} \mathbf{T}^{1 / 2}$, where $\mathbf{T} \in \mathbb{C}^{N_{c} \times N_{c}}$ is the subcarrier correlation matrix.

Proposition 5: The union bound on the symbol error probability of the ED-SSS operating under correlated channel condition is given by

$$
\mathrm{P}_{\mathrm{e}} \leq \frac{\left|\mathcal{C}_{k^{*}}\right|-1}{2}\left(\frac{\rho \bar{\lambda}^{*}}{4 n N_{a}}\right)^{-\bar{q} N_{r}},
$$

where $\bar{q}=\operatorname{rank}\left(\mathbf{X}_{\min } \mathbf{X}_{\text {min }}^{H} \mathbf{T}\right)$ and $\bar{\lambda}^{*}$ is the smallest nonzero Eigenvalue of $\mathbf{X}_{\min } \mathbf{X}_{\min }^{H} \mathbf{T}$.

The proof is along the same lines as that of Proposition 2, hence it is omitted. It is evident from Proposition 5 that a fullrank $\mathbf{T}$ is sufficient for attaining the diversity gain of $N_{c}-$ $N_{I M}+1$ and $N_{c}$ in case of ED-SSS and TPC-aided ED-SSS, respectively.

\section{Simulation Studies}

Simulation scenario: While evaluating a BER of $10^{-t}$, we have employed at least $10^{t+1}$ bits in all our simulations. The receiver is assumed to have perfect CSI and employ ML decoding in all the schemes considered. Furthermore, we note that the TPC matrix introduced in Sec. II.C may invoke any of the richly documented historical full-rank constructions in the space-time domain such as the lattice-based [62]-[64], field extension aided [65] and division algebras assited [66][68] constructions. However, it was astutely pointed out in [66] that the TPC matrices that achieve the optimal diversitymultiplexing tradeoff do not always lead to the best BER performance. Given that the existing TPC solutions conceived for OFDM-IM [50],[51],[43] are not generally applicable, based on our simulations, we opt for using the following rotation matrices contructed based on rotated $\mathbf{Z}^{n}$ lattices [62][64]. For the IM system associated with $N_{I M}=3$, we have

$$
\mathbf{P}=\left[\begin{array}{rrr}
-0.3279 & -0.5910 & -0.7369 \\
-0.7369 & -0.3279 & 0.5910 \\
-0.5910 & 0.7369 & -0.3279
\end{array}\right]
$$

and for $N_{I M}=4$, we have

$$
\mathbf{P}=\left[\begin{array}{rrrr}
-0.3663 & -0.7677 & 0.4230 & 0.3120 \\
-0.2264 & -0.4744 & -0.6845 & -0.5049 \\
-0.4744 & 0.2264 & -0.5049 & 0.6845 \\
-0.7677 & 0.3663 & 0.3120 & -0.4230
\end{array}\right]
$$

The correlated subcarriers are simulated by assuming $\mathbf{T}(i, j)=\zeta^{|i-j|}$ in [64], where $0 \leq \zeta \leq 1$ models the correlation coefficient. In our simulation studies, we consider two values of $\zeta \in\{0.1,0.9\}$, where 0.1 corresponds to a fairly uncorrelated channel scenario and 0.9 corresponds to a highcorrelation scenario.

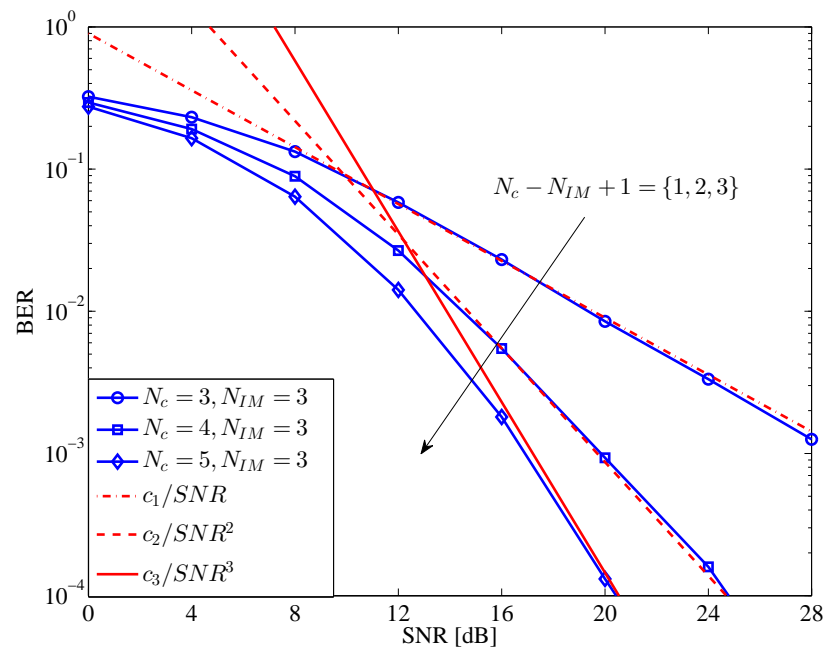

Fig. 1. BER performance of ED-SSS in an IM system having $N_{I M}=3$, $N_{a}=2, N_{r}=1, N_{c} \in\{3,4,5\}$ and employing 4-QAM signal set. The reference curves $c_{k} / S N R^{k}$ for $k \in\{1,2,3\}$ are provided to illustrate the attainable diversity order.

\section{A. Validation of Transmit Diversity Order of ED-SSS}

In order to validate the theoretical claims concerning the achievable diversity gain of ED-SSS, let us consider an IM system having $N_{I M}=3, N_{a}=2, N_{r}=1, N_{c} \in\{3,4,5\}$ and employing 4-QAM signal set. Fig. 1 depicts the attainable BER performance of ED-SSS for various values of $N_{c}$ as well as the reference curves $c_{k} / S N R^{k}$ for $k \in\{1,2,3\}$. The constants associated with the reference curves are given by $c_{1}=0.9, c_{2}=8.7$ and $c_{3}=145$. It is evident from Fig. 1 that the ED-SSS attains a transmit diversity order of $N_{c}-N_{I M}+1$. Fig. 2 depicts the attainable BER performance of ED-SSS in the aforementioned system when employing $N_{r}=2$. The reference curves $c_{k}^{\prime} / S N R^{k}$ for $k \in\{2,4,6\}$ are also provided for illustrating the attainable diversity order. The constants associated with the reference curves are given by $c_{1}^{\prime}=2.6, c_{2}^{\prime}=185$ and $c_{3}^{\prime}=29500$. It is evident from Fig. 2 that ED-SSS attains a total diversity order of $N_{r}\left(N_{c}-N_{I M}+1\right)=2\left(N_{c}-N_{I M}+1\right)$. Fig. 3 compares the attainable BER performance of ED-SSS in an IM system having $N_{I M}=4, N_{a}=2, N_{r} \in\{1,2\}, N_{c} \in\{4,5,6\}$ and employing 4-QAM signal set. Observe from Fig. 3 that EDSSS attains higher diversity gain when $N_{c}$ is increased from 4 to 6 .

\section{B. Performance of ED-SSS with Various Signal Sets}

Fig. 4 depicts the BER performance of ED-SSS in an IM system having $N_{I M}=3, N_{a}=2, N_{r}=1, N_{c} \in\{3,4\}$ and employing BPSK, 4-QAM and 8-QAM signal sets. It is seen from Fig. 4 that ED-SSS attains a higher diversity order for all the signal sets considered, when $N_{c}$ is increased. Similarly, Fig. 5 depicts the BER performance of ED-SSS in an IM system having $N_{I M}=4, N_{a}=2, N_{r}=1, N_{c} \in\{4,5\}$ and employing BPSK, 4-QAM and 8-QAM signal sets. Again, it is observed that ED-SSS attains a higher diversity order for 


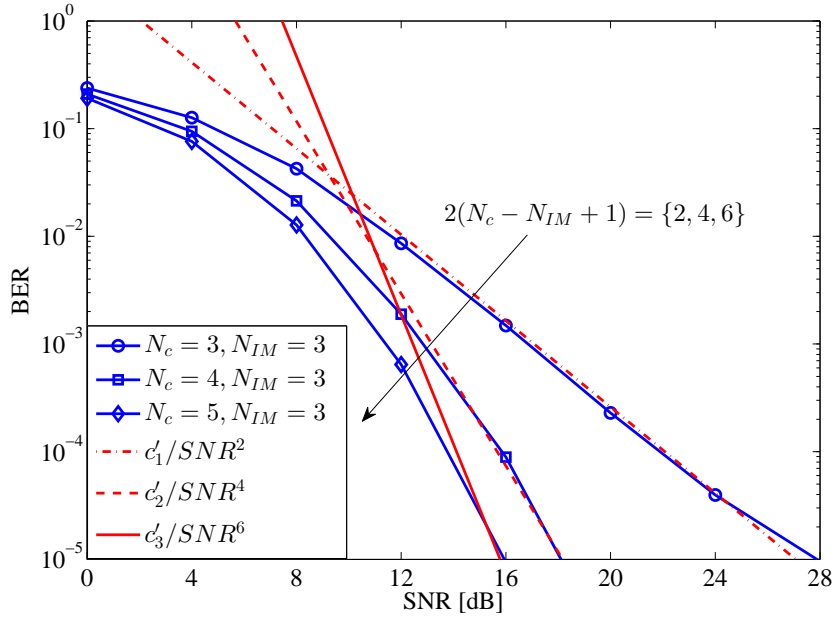

Fig. 2. BER performance of ED-SSS in an IM system having $N_{I M}=3$, $N_{a}=2, N_{r}=2, N_{c} \in\{3,4,5\}$ and employing 4-QAM signal set. The reference curves $c_{k}^{\prime} / S N R^{2 k}$ for $k \in\{1,2,3\}$ are provided to illustrate the attainable diversity order.
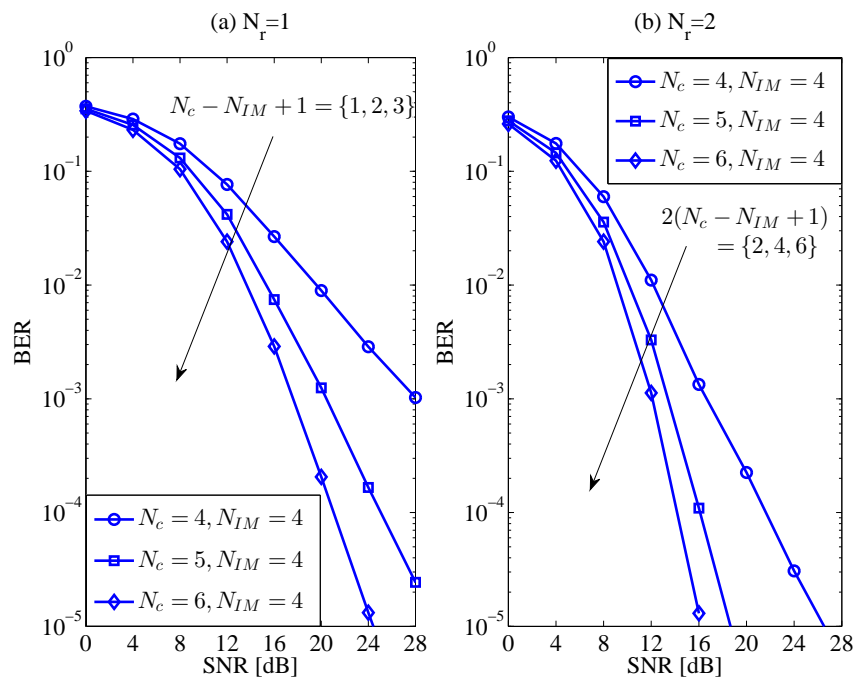

Fig. 3. BER performance of ED-SSS in an IM system having $N_{I M}=4$, $N_{a}=2, N_{r} \in\{1,2\}, N_{c} \in\{4,5,6\}$ and employing 4-QAM signal set. Plot (a) corresponds to $N_{r}=1$ and plot (b) corresponds to $N_{r}=2$.

all the signal sets considered, when $N_{c}$ is increased. Thus, we conclude that our results concerning the attainable diversity order of ED-SSS stands validated.

\section{Performance of ED-SSS with Precoding}

Fig. 6 compares the BER performance of TPC-aided EDSSS in an IM system having $N_{I M}=3, N_{a}=2, N_{r} \in\{1,2\}$, $N_{c} \in\{3,4,5\}$ and employing a 4-QAM signal set. It is evident from Fig. 6 that there is a significant performance improvement, when $N_{c}$ is increased. Similarly, Fig. 7 compares the BER performance of TPC-aided ED-SSS in an IM system having $N_{I M}=4, N_{a}=2, N_{r} \in\{1,2\}$, $N_{c} \in\{4,5,6\}$ and employing a 4-QAM signal set. Fig. 8 compares the BER performance of TPC-aided ED-SSS to that
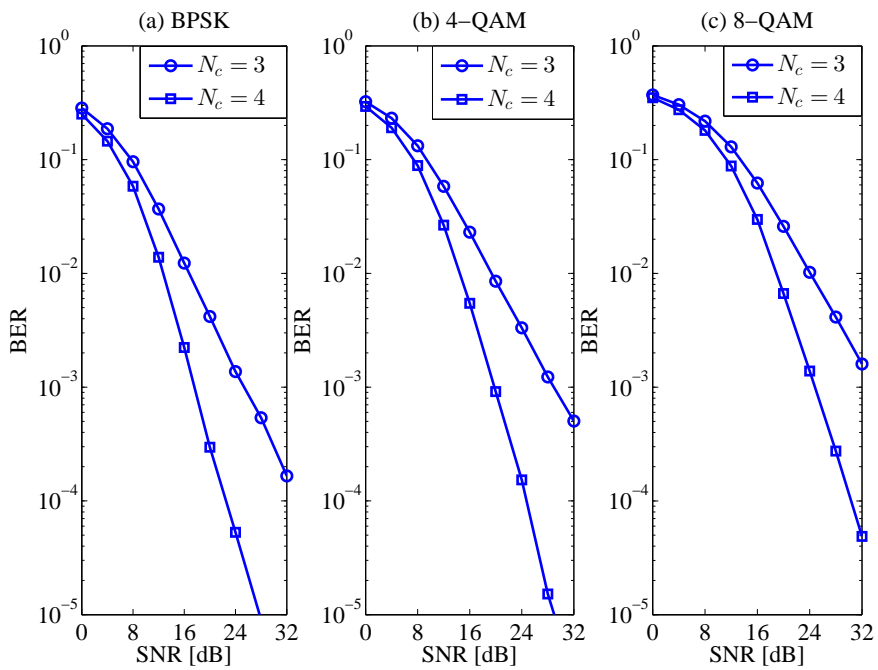

Fig. 4. BER performance of ED-SSS in an IM system having $N_{I M}=3$, $N_{a}=2, N_{r}=1, N_{c} \in\{3,4\}$ and employing BPSK, 4-QAM and 8-QAM signal sets. Plot (a), plot (b), and plot (c) correspond to BPSK, 4-QAM, and 8-QAM signal sets, respectively.
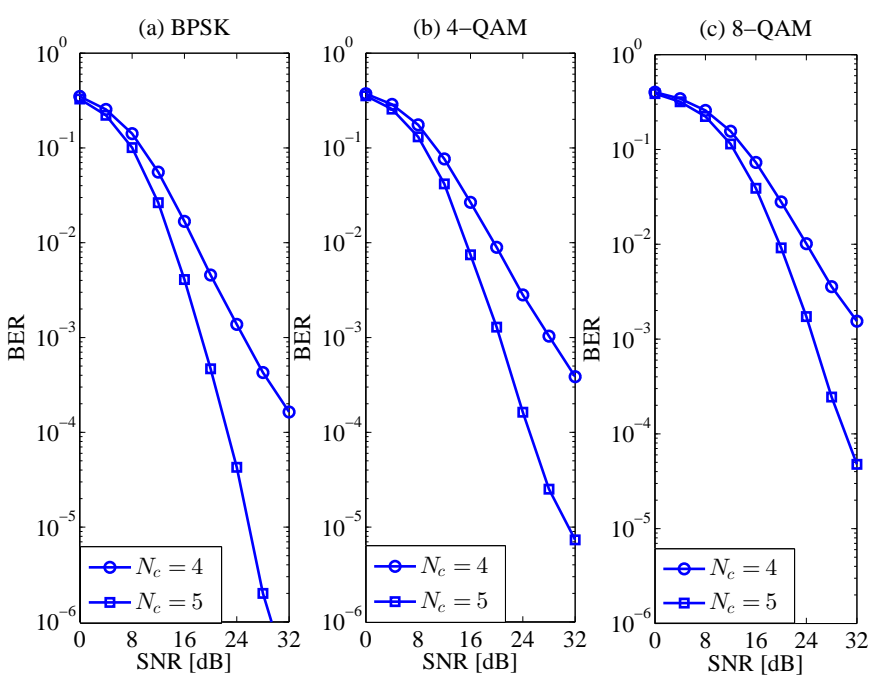

Fig. 5. BER performance of ED-SSS in an IM system having $N_{I M}=4$, $N_{a}=2, N_{r}=1, N_{c} \in\{4,5\}$ and employing BPSK, 4-QAM and 8-QAM signal sets. Plot (a), plot (b), and plot (c) correspond to BPSK, 4-QAM, and 8-QAM signal sets, respectively.

without precoding in an IM system having $N_{I M}=3, N_{a}=2$, $N_{r}=1, N_{c} \in\{3,5\}$ and employing a 4-QAM signal set. It is seen in Fig. 8 that TPC provides significant performance improvements over its counterpart operating without TPC. Specifically, at a BER of $10^{-3}$ an SNR gain of about $8 \mathrm{~dB}$ is observed, when $N_{c}=3$ and $N_{r}=1$. Furthermore, at a BER of $10^{-4}$, an SNR gain of about $5 \mathrm{~dB}$ is recorded for $N_{c}=3$ and $N_{r}=2$. When $N_{c}=5$, we see that TPC does not yield significant performance gains when $N_{I M}=3$. Thus, we may resort to ED-SSS dispensing with precoding when $N_{c}$ is large, whose complexity is significantly lower than that of its counterpart with precoding. However, when $N_{I M}=4$ 

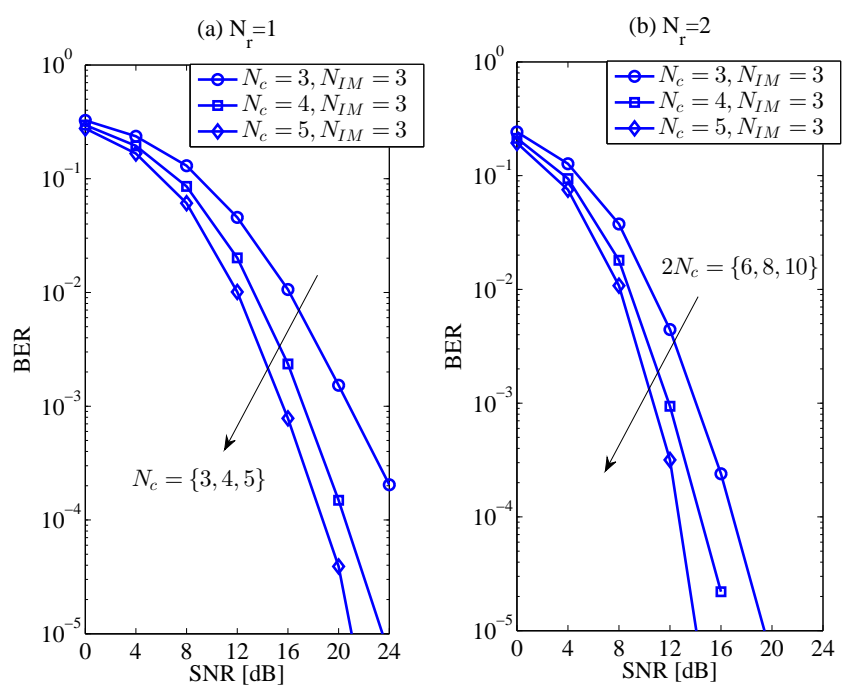

Fig. 6. BER performance of ED-SSS with precoding in an IM system having $N_{I M}=3, N_{a}=2, N_{c} \in\{3,4,5\}$ and employing 4-QAM signal set. Plot (a) and plot (b) correspond to $N_{r}=1$ and $N_{r}=2$, respectively.
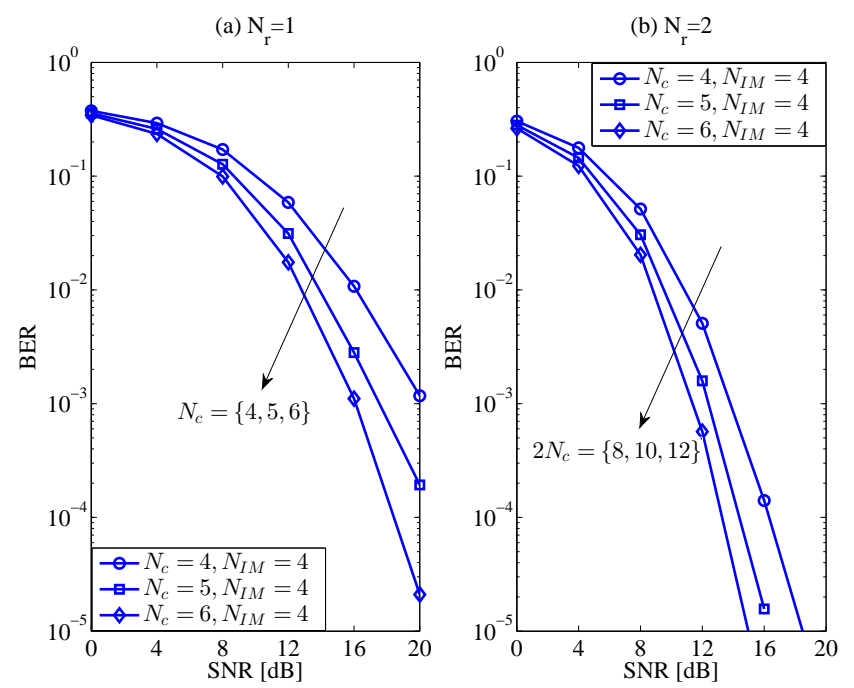

Fig. 7. BER performance of ED-SSS with precoding in an IM system having $N_{I M}=4, N_{a}=2, N_{c} \in\{4,5,6\}$ and employing 4-QAM signal set. Plot (a) and plot (b) correspond to $N_{r}=1$ and $N_{r}=2$, respectively.

we see that TPC does yield a significant performance gain. Specifically, at a BER of $10^{-5}$, we see an SNR gain of about $3 \mathrm{~dB}$, which is evident from Fig. 9(a).

\section{Performance under Correlated Subcarriers}

Fig. 10 compares the BER performance of ED-SSS in an IM system having $\left(N_{c}, N_{I M}\right) \in\{(5,3),(6,4)\}, N_{a}=2$, $N_{r}=1$, and employing a 4-QAM signal set, when operating in a correlated channel having $\zeta \in\{0.1,0.9\}$. Specifically, Fig. 10(a) and Fig. 10(b) correspond to $\left(N_{c}, N_{I M}\right)=(5,3)$ and $\left(N_{c}, N_{I M}\right)=(6,4)$, respectively. Observe from both the plots that the channel's correlation imposes significant performance degradations. Specifically, an SNR degradation of about $3 \mathrm{~dB}$ is observed at a BER of about $10^{-4}$, when the correlation
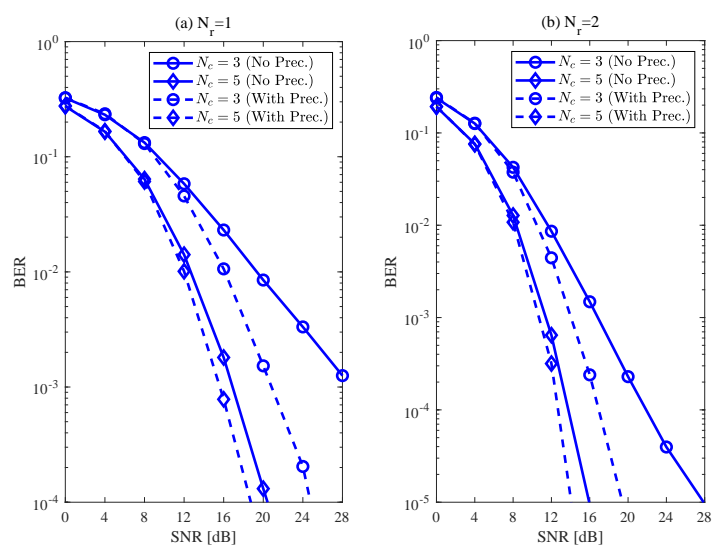

Fig. 8. Comparison of BER performance of ED-SSS with precoding with that without precoding in an IM system having $N_{I M}=3, N_{a}=2, N_{r}=$ $1, N_{c} \in\{3,5\}$ and employing 4-QAM signal set. Plot (a) and plot (b) correspond to $N_{r}=1$ and $N_{r}=2$, respectively.
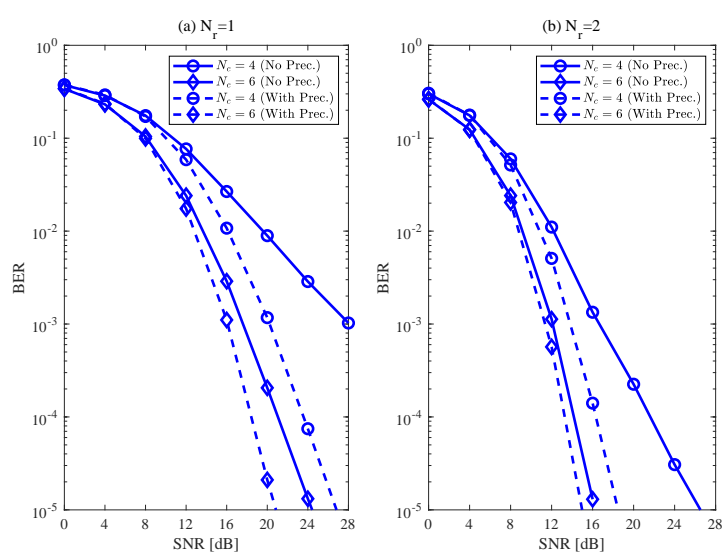

Fig. 9. Comparison of BER performance of ED-SSS with precoding with that without precoding in an IM system having $N_{I M}=4, N_{a}=2, N_{r}=$ $1, N_{c} \in\{4,6\}$ and employing 4-QAM signal set. Plot (a) and plot (b) correspond to $N_{r}=1$ and $N_{r}=2$, respectively.

factor $\zeta$ is increased from 0.1 to 0.9 . Similar observations also hold for the TPC-aided ED-SSS, which is evident from Fig. 11. Although there is a gradual performance degradation when the channel's correlation factor is increased, it is evident from both Fig. 10 and Fig. 11 that the proposed schemes retains the diversity gain guaranteed by our theoretical analysis.

\section{CONCLUSions}

We have considered a subcarrier IM aided system and proposed Euclidean distance based subcarrier subset selection in order to increase the diversity gain. We have quantified the attainable transmit diversity order of ED-SSS and shown that to be amenable to low-complexity implementation. Furthermore, we have extended ED-SSS with the aid of precoding, which was shown to overcome the limitation of ED-SSS by attaining full transmit diversity gain. Simulation results were presented to validate the theoretical claims and also to demonstrate the performance gains of the proposed schemes. 

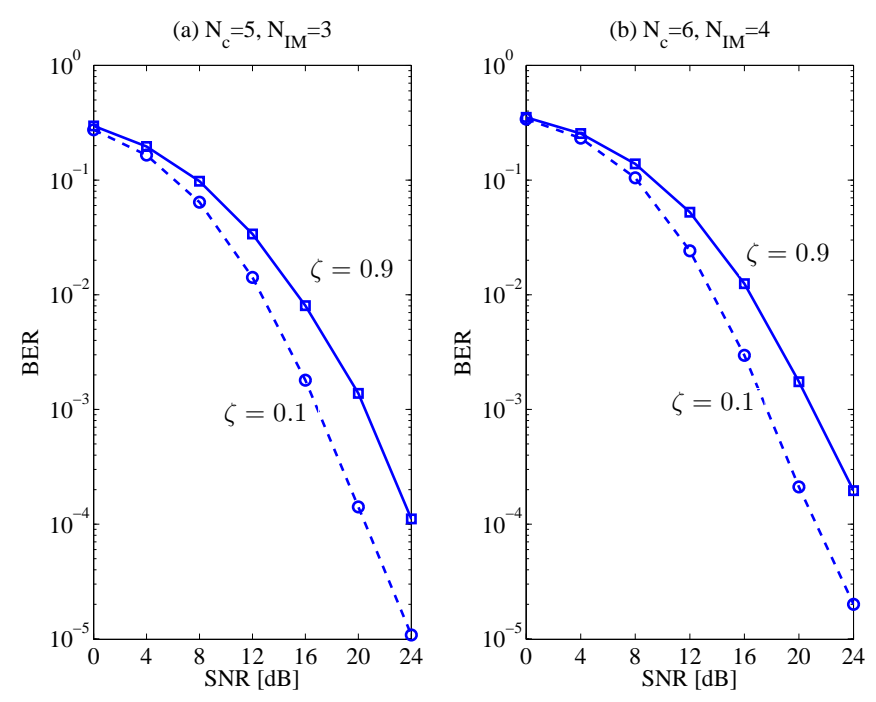

Fig. 10. Comparison of BER performance of ED-SSS in an IM system having $N_{I M} \in\{3,4\}, N_{c} \in\{5,6\}, N_{a}=2, N_{r}=1$, and employing 4-QAM signal set while operating in a correlated channel having $\zeta \in\{0.1,0.9\}$.
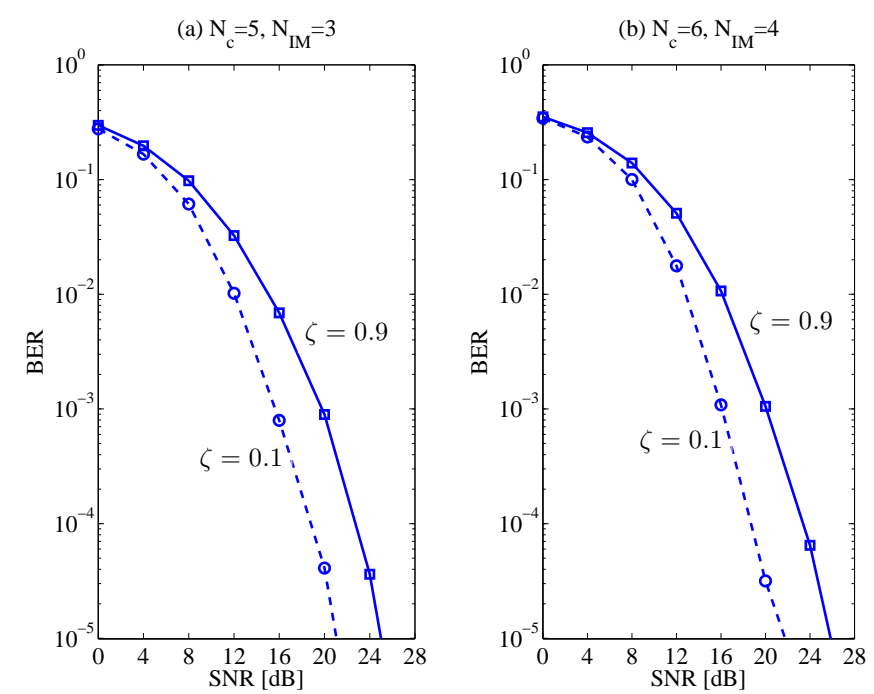

Fig. 11. Comparison of BER performance of ED-SSS with precoding in an IM system having $N_{I M} \in\{3,4\}, N_{c} \in\{5,6\}, N_{a}=2, N_{r}=1$, and employing 4-QAM signal set while operating in a correlated channel having $\zeta \in\{0.1,0.9\}$.

\section{PROOF OF PROPOSITION 1}

Proof: The problem of finding the optimal subcarrier subset in (4) can be equivalently written as

$$
\begin{aligned}
I^{*} & =\arg \max _{I \in \mathcal{I}} \min _{\mathbf{z} \in \Delta \mathcal{X}}\left\|\mathbf{H}_{I} \mathbf{z}\right\|^{2}, \\
& =\arg \max _{I \in \mathcal{I}} \min _{\mathbf{z} \in \Delta \mathcal{X}} \sum_{i \in I}\left\|\mathbf{h}_{i} \mathbf{z}(i)\right\|^{2}, \\
& =\arg \max _{I \in \mathcal{I}} \min _{\mathbf{z} \in \Delta \mathcal{X}} \sum_{i \in I}\left\|\mathbf{h}_{i}\right\|^{2}|\mathbf{z}(i)|^{2} .
\end{aligned}
$$

Any $\mathbf{z} \in \Delta \mathcal{X}$ can be expressed as $\mathbf{z}=\sum_{i=1}^{N_{a}} s_{i} \mathbf{e}_{j_{i}}$ $\sum_{i=1}^{N_{a}} s_{i}^{\prime} \mathbf{e}_{k_{i}}$, where $Q_{1}=\left\{j_{1}, j_{2}, \ldots, j_{N_{a}}\right\}$ and $Q_{2}=$ $\left\{k_{1}, k_{2}, \ldots, k_{N_{a}}\right\}$ are the support sets of the transmit vectors associated with $\mathbf{z}, s_{i}$ and $s_{i}^{\prime}$ are $M$-QAM symbols. Let $Q^{c}=Q_{1} \cap Q_{2}, Q_{1}^{d}=Q_{1} \backslash Q^{c}$ and $Q_{2}^{d}=Q_{2} \backslash Q^{c}$. Note that $0 \leq\left|Q^{c}\right| \leq N_{a}$. Furthermore, we have

$$
|\mathbf{z}(i)|^{2}= \begin{cases}\left|s_{i}-s_{i}^{\prime}\right|^{2}, & i \in Q^{c} \\ \left|s_{i}\right|^{2}, & i \in Q_{1}^{d} . \\ \left|s_{i}^{\prime}\right|^{2}, & i \in Q_{2}^{d}\end{cases}
$$

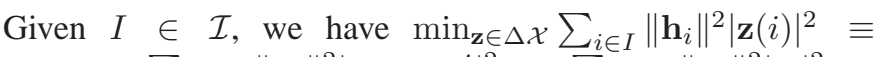
$\min _{\mathbf{z} \in \Delta \mathcal{X}} \sum_{i \in Q^{c}}\left\|\mathbf{h}_{i}\right\|^{2}\left|s_{i}-s_{i}^{\prime}\right|^{2}+\sum_{i \in Q_{1}^{d}}\left\|\mathbf{h}_{i}\right\|^{2}\left|s_{i}\right|^{2}+$ $\sum_{i \in Q_{2}^{d}}\left\|\mathbf{h}_{i}\right\|^{2}\left|s_{i}^{\prime}\right|^{2}$. Let the elements of $I$ be arranged as $\left\{m_{1}, m_{2}, \ldots, m_{N_{I M}}\right\}$ such that $\left\|\mathbf{h}_{m_{1}}\right\|^{2}>\left\|\mathbf{h}_{m_{2}}\right\|^{2}>$ $\cdots>\left\|\mathbf{h}_{m_{N_{I M}}}\right\|^{2}$. When $\left|Q^{c}\right|=N_{a}$, we have $\left|Q_{1}^{d}\right|=$ $\left|Q_{2}^{d}\right|=0$ and $\min _{\mathbf{z} \in \Delta \mathcal{X}} \sum_{i \in I}\left\|\mathbf{h}_{i}\right\|^{2}|\mathbf{z}(i)|^{2}=4\left\|\mathbf{h}_{m_{N_{I M}}}\right\|^{2}$, which follows from the fact that $\min _{\mathbf{z} \in \Delta \mathcal{X} \sum_{i \in I}|\mathbf{z}(i)|^{2}=}$ 4 when a regular $M$-QAM constellation is employed. When $\left|Q^{c}\right|=N_{a}-1$, we have $\left|Q_{1}^{d}\right|=\left|Q_{2}^{d}\right|=$ 1 and $\min _{\mathbf{z} \in \Delta \mathcal{X}} \sum_{i \in I}\left\|\mathbf{h}_{i}\right\|^{2}|\mathbf{z}(i)|^{2}=2\left\|\mathbf{h}_{m_{N_{I M}-1}}\right\|^{2}+$ $2\left\|\mathbf{h}_{m_{N_{I M}}}\right\|^{2}>4\left\|\mathbf{h}_{m_{N_{I M}}}\right\|^{2}$. It is straightforward to show that for $\left|Q^{c}\right|<N_{a}-1$, we have $\min _{\mathbf{z} \in \Delta \mathcal{X}} \sum_{i \in I}\left\|\mathbf{h}_{i}\right\|^{2}|\mathbf{z}(i)|^{2}>$ $4\left\|\mathbf{h}_{m_{N_{I M}}}\right\|^{2}$. Thus, when employing a regular $M$-QAM, we have $\min _{\mathbf{z} \in \Delta \mathcal{X}} \sum_{i \in I}\left\|\mathbf{h}_{i}\right\|^{2}|\mathbf{z}(i)|^{2}=4\left\|\mathbf{h}_{m_{N_{I M}}}\right\|^{2}$. Thus, the

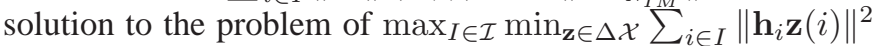
can be obtained by successively eliminating the subsets that are associated with the subcarriers $l_{N_{c}}, l_{N_{c}-1}, \ldots, l_{N_{I M}+1}$, which yields $I^{*}=\left\{l_{1}, l_{2}, \ldots, l_{N_{I M}}\right\}$.

\section{ACKNOWLEDGEMENTS}

The authors acknowledge the use of the IRIDIS High Performance Computing Facility, and associated support services at the University of Southampton, in the completion of this work.

\section{REFERENCES}

[1] R. Mesleh, H. Haas, S. Sinanovic, C. Ahn and S. Yun "Spatial modulation," IEEE Trans. Veh. Technol., vol. 57, no. 4, pp. 2228-2242, July 2008.

[2] M. Di Renzo, H. Haas and P. M. Grant, "Spatial modulation for multipleantenna wireless systems - A survey," IEEE Commun. Magazine, vol. 49, no. 12, pp. 182-191, Dec. 2011.

[3] M. Di Renzo, H. Haas, A. Ghrayeb, S. Sugiura and L. Hanzo, "Spatial modulation for generalized MIMO: challenges, opportunities, and implementation," Proceedings of the IEEE, vol. 102, no. 1, pp. 56-103, Jan. 2014

[4] A. Stavridis, S. Sinanovic, M. Di Renzo and H. Haas, "Energy evaluation of spatial modulation at a multi-antenna base station," 2013 IEEE 78th Vehicular Technology Conference (VTC Fall), Las Vegas, NV, 2013. 
[5] X. Cheng, M. Zhang, M. Wen and L. Yang, "Index modulation for 5G: Striving to do more with less," IEEE Wireless Commun., vol. 25, no. 2 pp. 126-132, April 2018.

[6] X. Cheng, M. Wen and L. Yang, "Index modulation for 5G wireless communications," Springer International Publishing, 2017.

[7] C. Xu, S. Sugiura, S. X. Ng, P. Zhang, L. Wang and L. Hanzo, "Two decades of MIMO design tradeoffs and reduced-complexity MIMO detection in near-capacity systems," IEEE Access, vol. 5, pp. 1856418632, 2017.

[8] I. E. Telatar "Capacity of multi-antenna Gaussian channels," Eur. Trans. Telecommun., vol. 10, no. 6, pp. 585-595, Dec. 1999.

[9] P. Wolniansky, G. Foschini, G. Golden and R. Valenzuela, "V-BLAST: an architecture for realizing very high data rates over the rich-scattering wireless channel," in Proc. International Symp. Signals, Syst., Electron., Pisa, Italy, pp. 295-300, Sep. 1998.

[10] C. Xu, P. Zhang, R. Rajashekar, N. Ishikawa, S. Sugiura, L. Wang and L. Hanzo , "Finite-cardinality single-RF differential space-time modulation for improving the diversity-throughput tradeoff," IEEE Trans. Commun., vol. 67, no. 1, pp. 318-335, Jan. 2019.

[11] C. Xu, R. Rajashekar, N. Ishikawa, S. Sugiura and L. Hanzo, "SingleRF index shift keying aided differential space-time block coding," IEEE Trans. Sig. Proc., vol. 66, no. 3, pp. 773-788, Feb. 2018.

[12] R. Rajashekar, K.V.S. Hari, L. Hanzo, "Reduced-complexity ML detection and capacity-optimized training for spatial modulation systems," IEEE Trans. Commun., vol. 62, no. 1, pp. 112-125, Jan. 2014.

[13] C. Xu, S. Sugiura, S. X. Ng and L. Hanzo, "Spatial modulation and space-time shift keying: optimal performance at a reduced detection complexity," IEEE Trans. Commun., vol. 61, no. 1, pp. 206-216, January 2013.

[14] P. Som and A. Chockalingam, "Spatial modulation and space shift keying in single carrier communication," IEEE 23rd International Symposium on Personal, Indoor and Mobile Radio Communications (PIMRC), Sydney, NSW, pp. 1962-1967, 2012.

[15] R. Rajashekar, K.V.S. Hari and L. Hanzo, "Spatial modulation aided zero-padded single carrier transmission for dispersive channels," IEEE Trans. Commun., vol. 61, no. 6, pp. 2318-2329, June 2013.

[16] L. He, J. Wang and J. Song, "Information-aided iterative equalization: A novel approach for single-carrier spatial modulation in dispersive channels," IEEE Trans. Veh. Technol., vol. 66, no. 5, pp. 4448-4452, May 2017.

[17] E. Basar, U. Aygolu, E. Panayirci and H. V. Poor, "Space-time block coding for spatial modulation," IEEE Trans. Commun., vol. 59, no. 3 , pp. 823-832, Mar. 2011.

[18] R. Rajashekar and K.V.S. Hari, "Modulation diversity for spatial modulation using complex interleaved orthogonal design," in Proc. IEEE TENCON 2012, Nov. 2012, pp. 1-6.

[19] X. Li and L. Wang, "High rate space-time block coded spatial modulation with cyclic structure," IEEE Commun. Lett., vol. 18, no. 4, pp. 532-535, Apr. 2014

[20] A. G. Helmy, M. Di Renzo and N. Al-Dhahir, "Enhanced-reliability cyclic generalized spatial-and-temporal modulation," IEEE Commun. Lett., vol. 20, no. 12, pp. 2374-2377, Dec. 2016.

[21] C. Xu, P. Zhang, R. Rajashekar, N. Ishikawa, S. Sugiura, Z. Wang and L. Hanzo, "“Near-perfect" finite-cardinality generalized space-time shift keying," IEEE J. Sel. Areas Commun. (accepted).

[22] C. Xu, T. Bai, J. Zhang, R.G. Maunder, S. Sugiura, Z. Wang and L. Hanzo, "Constant-envelope space-time shift keying," IEEE J. Sel. Topics Signal Process. (accepted).

[23] P. Yang, Y. Xiao, L. Li, Q. Tang, Y. Yu and S. Li, "Link adaptation for spatial modulation with limited feedback," IEEE Trans. Veh. Technol., vol. 61, no. 8, pp. 3808-3813, Oct. 2012

[24] R. Rajashekar, K.V.S. Hari and L. Hanzo, "Antenna selection in spatial modulation systems," IEEE Commun. Lett., vol. 17, no. 3, pp. 521-524, Mar. 2013.

[25] R. Rajashekar, K.V.S. Hari, K. Giridhar and L. Hanzo, "Performance analysis of antenna selection algorithms in spatial modulation systems with imperfect CSIR," European Wireless 2013; 19th European Wireless Conference, Guildford, UK, 2013, pp. 1-5.

[26] R. Rajashekar, K.V.S. Hari and L. Hanzo, "Quantifying the transmit diversity order of Euclidean distance based antenna selection in spatial modulation," IEEE Signal Proc. Lett., vol. 22, no. 9, pp. 1434-1437, Sept. 2015.

[27] R. Rajashekar, L. Yang, K.V.S. Hari and L. Hanzo, "Transmit antenna subset selection in generalised spatial modulation systems," to appear in IEEE Trans. Veh. Technol., doi: 10.1109/TVT.2018.2889024.
[28] Z. Zhou, N. Ge and X. Lin, "Reduced-complexity antenna selection schemes in spatial modulation," IEEE Commun. Lett., vol. 18, no. 1, pp. 14-17, Jan. 2014.

[29] N. Wang, W. Liu, H. Men, M. Jin, and H. Xu, "Further complexity reduction using rotational symmetry for EDAS in spatial modulation," IEEE Commun. Lett., vol. 18, no. 10, pp. 1835-1838, Oct. 2014.

[30] Z. Sun, Y. Xiao, L. You, L. Yin, P. Yang and S. Li, "Cross-entropy-based antenna selection for spatial modulation," IEEE Commun. Lett., vol. 20, no. 3, pp. 622-625, March 2016.

[31] P. Yang, Y. Xiao, Y. L. Guan, S. Li and L. Hanzo, "Transmit antenna selection for multiple-input multiple-output spatial modulation systems," IEEE Trans. Commun., vol. 64, no. 5, pp. 2035-2048, May 2016.

[32] Z. Sun, Y. Xiao, P. Yang, S. Li and W. Xiang, "Transmit antenna selection schemes for spatial modulation systems: search complexity reduction and large-scale MIMO applications," IEEE Trans. Veh. Technol. vol. 66, no. 9, pp. 8010-8021, Sep. 2017.

[33] Y. Naresh and A. Chockalingam, "On media-based modulation using RF mirrors," IEEE Trans. Veh. Technol. vol. 66, no. 6, pp. 4967-4983, June 2017.

[34] R. Abu-alhiga and H. Haas, "Subcarrier-index modulation OFDM," 2009 IEEE 20th International Symposium on Personal, Indoor and Mobile Radio Communications, Tokyo, 2009, pp. 177-181.

[35] D. Tsonev, S. Sinanovic and H. Haas, "Enhanced subcarrier index modulation (SIM) OFDM," 2011 IEEE GLOBECOM Workshops (GC Wkshps), Houston, TX, 2011, pp. 728-732.

[36] E. Basar, U. Aygolu, E. Panayirci and H. V. Poor, "Orthogonal frequency division multiplexing with index modulation," IEEE Trans. Sig. Proc., vol. 61, no. 22, pp. 5536-5549, Nov. 2013.

[37] E. Basar, "On multiple-input multiple-output OFDM with index modulation for next generation wireless networks," IEEE Trans. Sig. Proc., vol. 64, no. 15 , pp. 3868-3878, Aug. 2016.

[38] N. Ishikawa, S. Sugiura and L. Hanzo, "Subcarrier-index modulation aided OFDM - Will it work?," IEEE Access, vol. 4, no. , pp. 25802593, 2016

[39] E. Basar, M. Wen, R. Mesleh, M. Di Renzo, Y. Xiao and H. Haas, "Index modulation techniques for next-generation wireless networks," IEEE Access, vol. 5, pp. 16693-16746, 2017.

[40] J. Zheng and H. Lv, "Peak-to-average power ratio reduction in OFDM index modulation through convex programming," IEEE Commun. Lett. vol. 21, no. 7, pp. 1505-1508, July 2017.

[41] P. Yang, Y. Xiao, Y. L. Guan, M. Di Renzo, S. Li and L. Hanzo, "Multidomain index modulation for vehicular and railway communications: a survey of novel techniques," IEEE Veh. Technol. Mag., vol. 13, no. 3, pp. 124-134, Sept. 2018.

[42] E. Basar, "OFDM With index modulation using coordinate interleaving," IEEE Wireless Commun. Lett., vol. 4, no. 4, pp. 381-384, Aug. 2015.

[43] Q. Li, M. Wen, E. Basar, H.V. Poor, B. Zheng and F. Chen, "Diversity enhancing multiple-mode OFDM with index modulation," IEEE Trans. Commun., vol. 66, no. 8, pp. 3653-3666, Aug. 2018.

[44] M. Wen, E. Basar, Q. Li, B. Zheng and M. Zhang, "Multiple-mode orthogonal drequency division multiplexing with index modulation," IEEE Trans. Commun., vol. 65, no. 9, pp. 3892-3906, Sept. 2017.

[45] S. Dang, G. Chen and J.P. Coon, "Lexicographic codebook design for OFDM with index modulation," IEEE Trans. Wireless Commun., vol. 17, no. 12, pp. 8373-8387, Dec. 2018.

[46] J. Zheng, "Adaptive index modulation for parallel Gaussian channels with finite alphabet inputs," IEEE Trans. Veh. Technol., vol. 65, no. 8, pp. 6821-6827, Aug. 2016.

[47] S. Dang, J.P. Coon and G. Chen, "Adaptive OFDM with index modulation for two-hop relay-assisted networks," IEEE Trans. Wireless Commun., vol. 17, no. 3, pp. 1923-1936, March 2018

[48] J. Choi, "Coded OFDM-IM with transmit diversity," IEEE Trans. Commun., vol. 65, no. 7, pp. 3164-3171, July 2017.

[49] J. Zheng and R. Chen, "Achieving transmit diversity in OFDM-IM by utilizing multiple signal constellations," IEEE Access, vol. 5, no. , pp 8978-8988, May 2017.

[50] S. Gao, M. Zhang and X. Cheng, "Precoded index modulation for multiinput multi-output OFDM," IEEE Trans. Wireless Commun., vol. 17, no. 1, pp. 17-28, Jan. 2018

[51] H. Zhang, C. Jiang, L. Yang, E. Basar and L. Hanzo, "Linear precoded index modulation,” IEEE Trans. Commun., vol. 67, no. 1, pp. 350-363, Jan. 2019.

[52] T.H. Cormen, C.E. Leiserson, R.L.Rivest and C. Stein, "Introduction to algorithms," The MIT press, 2001.

[53] M.A. Bender, M. Farach-Colton and M. Mosteiro, "Theory of computing systems," Insertion sort is O(nlogn), vol. 39, no. 3, pp. 391-397, 2019 
[54] C. Xu, X. Zuo, S.X. Ng, R.G. Maunder and L. Hanzo, "Reducedcomplexity soft-decision multiple-symbol differential sphere detection," IEEE Trans. Commun., vol. 63, no. 9, pp. 3275-3289, Sept. 2015.

[55] M. Wen, X. Cheng, M. Ma, B. Jiao and H.V. Poor, "On the achievable rate of OFDM with index modulation," IEEE Trans. Signal Process., vol. 64, no. 8, pp. 1919-1932, April, 2016.

[56] N. Ishikawa, S. Sugiura and L. Hanzo, "Subcarrier-index modulation aided OFDM - Will it work?," IEEE Access, vol. 4, pp. 2580-2593, 2016.

[57] D. Tsonev, S. Sinanovic and H. Haas, "Enhanced subcarrier index modulation (SIM) OFDM," IEEE GLOBECOM Workshops, 2001.

[58] R. Rajashekar, C. Xu, N. Ishikawa, L.L. Yang and L. Hanzo, "Multicarrier division duplex aided mm-Wave communications," IEEE Access (accepted).

[59] S. Dang, G. Ma, B. Shihada and M. Alouini, "A novel error performance analysis methodology for OFDM-IM," IEEE Wireless Commun. Lett., vol. 8, no. 3, pp. 897-900, June 2019.

[60] T.V. Luong and Y. Ko, "Spread OFDM-IM with precoding matrix and low-complexity detection designs," IEEE Trans. Veh. Technol., vol. 67, no. 12, pp. 11619-11626, Dec. 2018.

[61] D. Tse and P. Viswanath, "Fundamentals of wireless communications." Cambridge University Press, 2005.

[62] M. O. Damen, K. Abed-Meraim, and J. C. Belfiore, "Diagonal algebraic space-time block codes," IEEE Trans. Inf. Theory, vol. 48, no. 3, pp. 628-636, Mar. 2002.

[63] E. Bayer-Fluckiger, F. Oggier, and E. Viterbo, "New algebraic constructions of rotated Zn-lattice constellations for the Rayleigh fading channel," IEEE Trans. Inf. Theory, vol. 50, no. 4, pp. 702-714, Apr. 2004.

[64] E. Viterbo. Full Diversity Rotations, Mar. 16, 2005. [Online]. Available: http://www.ecse.monash.edu.au/staff/eviterbo/rotations/rotations.html

[65] M.O. Damen, A. Tewfik and J.C. Belflore, "A construction of a spacetime code based on number theory," IEEE Trans. Inf. Theory, vol. 48, no. 3, pp. 753-760, Mar. 2002.

[66] K.T., B.S. Rajan, "STBC-schemes with nonvanishing determinant for certain number of transmit antennas," IEEE Trans. Inf. Theory, vol. 51, no. 8, pp. 2984-2992, Aug. 2005.

[67] P. Elia, K.R. Kumar, S.A. Pawar, P.V. Kumar and H. Lu, "Explicit spacetime codes achieving the diversity-multiplexing gain tradeoff," IEEE Trans. on Inf. Theory, vol. 52, no. 9, pp. 3869-3884, 2006.

[68] F. Oggier, G. Rekaya, J. Belfiore and E. Viterbo, "Perfect space-time block codes," IEEE Trans. Inf. Theory, vol. 52, no. 9, pp. 3885-3902, Sept. 2006.

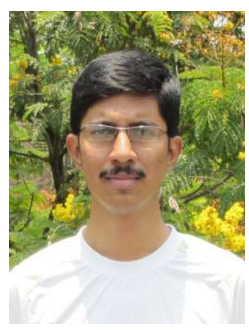

Rakshith Rajashekar (M'14-SM'17) received the B.E. degree in electrical communication engineering from Visvesvaraya Technological University, Karnataka, India, in 2007. He received his Ph.D. from the Department of Electrical Communication Engineering, Indian Institute of Science (IISc), India, in 2014. He is presently working as a Research Fellow at the University of Southampton, UK. Before joining the University of Southampton, he worked at Accord Software \& Systems, Bengaluru, India, as a Systems Engineer from 2007 to 2009, and as a Senior Scientist at Broadcom Communications, Bengaluru, India from 2014 to 2015. His research interests include antenna selection in MIMO systems, differential communication, millimeter wave communication, communication between drones with a focus on space-time signal processing and coding. $\mathrm{He}$ is the recipient of the Special Recognition award from Broadcom Communications, India and the Dean's Award from the University of Southampton, UK, for excellence in research. He has received the Best Reviewer award from IEEE Transactions on Wireless Communications, IEEE Transactions on Communications and IEEE Wireless Communications Letters.

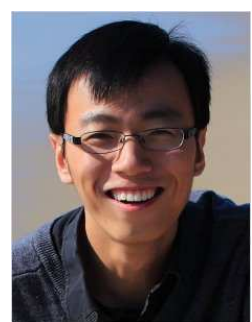

Chao Xu (S'09-M'14) received a B.Eng. degree from Beijing University of Posts and Telecommunications, China, and a BSc(Eng) with First Class Honours from Queen Mary, University of London, UK, through a Sino-UK joint degree program in 2008, both in Telecommunications Enginneering with Management. He obtained a MSc degree with distinction in Radio Frequency Communication Systems and a Ph.D. degree in Wireless Communications from the University of Southampton, UK in 2009 and 2015, respectively. He is currently a postdoctoral researcher working at Next Generation Wireless Research Group, University of Southampton, UK. His research interests include index modulation, reduced-complexity MIMO design, noncoherent detection and turbo detection. He was awarded the Best M.Sc. Student in Broadband and Mobile Communication Networks by the IEEE Communications Society (United Kingdom and Republic of Ireland Chapter) in 2009. He also received 2012 Chinese Government Award for Outstanding Self-Financed Student Abroad.

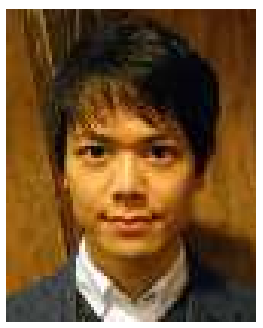

Naoki Ishikawa (S'13-M'17) was born in Kanagawa, Japan, in 1991. He received the B.E., M.E., and Ph.D. degrees from the Tokyo University of Agriculture and Technology, Tokyo, Japan, in 2014, 2015, and 2017, respectively. From June 2015 to September 2015, he was an academic visitor with the School of Electronics and Computer Science, University of Southampton, UK. From April 2016 to March 2017, he was a research fellow of the Japan Society for the Promotion of Science. From April 2017, he has been an assistant professor in the Graduate School of Information Sciences, Hiroshima City University, Japan.

He was certified as an Exemplary Reviewer of IEEE TRANSACTIONS ON COMMUNICATIONS 2017. He also received eight domestic awards, including the Yasujiro Niwa Outstanding Paper Award from Tokyo Denki University in 2018, the Telecom System Technology student Award (honorable mention) from Telecommunications Advancement Foundation of Japan in 2014, and the Outstanding Paper Award for Young C\&C Researchers from NEC C\&C Foundation in 2014.

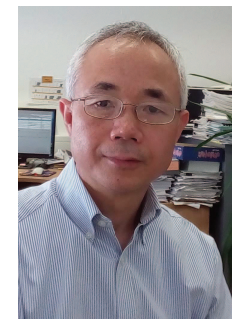

Lie-Liang Yang (M'98, SM'02, F'16) eceived his BEng degree in communications engineering from Shanghai TieDao University, Shanghai, China in 1988, and his MEng and PhD degrees in communications and electronics from Northern (Beijing) Jiaotong University, Beijing, China in 1991 and 1997, respectively. From June 1997 to December 1997 he was a visiting scientist to the Institute of Radio Engineering and Electronics, Academy of Sciences of the Czech Republic. Since December 1997, he has been with the University of Southampton, United Kingdom, where he is the professor of Wireless Communications in the School of Electronics and Computer Science. He has research interest in wireless communications, wireless networks and signal processing for wireless communications, as well as molecular communications and nano-networks. $\mathrm{He}$ has published over 370 research papers in journals and conference proceedings, authored/co-authored three books and also published several book chapters. The details about his research publications can be found at https://www.ecs.soton.ac.uk/people/llyang. He is a fellow of both the IEEE and the IET, and was a distinguished lecturer of the IEEE VTS. He served as an associate editor to the IEEE Trans. on Vehicular Technology and Journal of Communications and Networks (JCN), and is currently an associate editor to the IEEE Access and a subject editor to the IET Electronics Letters. 


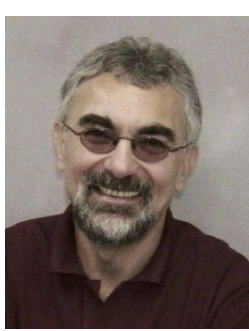

Lajos Hanzo (M'91-SM'92-F'04)

(http://www-mobile.ecs.soton.ac.uk) FREng,

FIEEE, FIET, Fellow of EURASIP, DSc received his degree in electronics in 1976 and his doctorate in 1983. In 2009 he was awarded an honorary doctorate by the Technical University of Budapest, while in 2015 by the University of Edinburgh. During his 40-year career in telecommunications he has held various research and academic posts in Hungary, Germany and the UK. Since 1986 he has been with the School of Electronics and Computer Science, University of Southampton, UK, where he holds the chair in telecommunications. He has successfully supervised about 100 $\mathrm{PhD}$ students, co-authored 20 John Wiley/IEEE Press books on mobile radio communications totalling in excess of 10000 pages, published $1500+$ research entries at IEEE Xplore, acted both as TPC and General Chair of IEEE conferences, presented keynote lectures and has been awarded a number of distinctions. Currently he is directing a 60 -strong academic research team, working on a range of research projects in the field of wireless multimedia communications sponsored by industry, the Engineering and Physical Sciences Research Council (EPSRC) UK, the European Research Council's Advanced Fellow Grant and the Royal Society's Wolfson Research Merit Award. He is an enthusiastic supporter of industrial and academic liaison and he offers a range of industrial courses. He is also a Governor of the IEEE VTS. During 2008 - 2012 he was the Editor-in-Chief of the IEEE Press and a Chaired Professor also at Tsinghua University, Beijing. His research is funded by the European Research Council's Senior Research Fellow Grant. For further information on research in progress and associated publications please refer to http://www-mobile.ecs.soton.ac.uk Lajos has 24 000 citations. 